Journal of Mathematics and Statistics 6 (2): 125-130, 2010

ISSN 1549-3644

(C) 2010 Science Publications

\title{
Integrability and Prolongation Structure of a Generalized Korteweg-de Vries Equation
}

\author{
Paul Bracken \\ Department of Mathematics, University of Texas, Edinburg, TX 78541-2999
}

\begin{abstract}
A closed differential ideal is constructed for a generalized Korteweg-de Vries equation. The ideal can be used to establish integrability for the equation. Some prolongation structures are determined for the equation and some larger prolongation algebras for given instances of the equation are found. The significance of this is that Backlund transformations can be developed based on them. It is shown how a Backlund transformation for a case of the equation can be formulated.
\end{abstract}

Key words: Differential ideal, prolongation, Backlund transformation, symmetry group

\section{INTRODUCTION}

The concept of integrability of certain kinds of partial differential equations or systems of such equations has generated a great deal of attention. There are many implications and related applications of the idea of integrability (Das, 1989). A Lax pair can be determined in theory, as well as an infinite number of conservation laws and perhaps more importantly, a Backlund transformation. A Backlund transformation has more important practical consequences, since it can be used to determine solutions to an associated equation, usually referred to as the potential equation, from solutions of the given equations. Of course, determining any of these in practice in a particular case is not easy, however, there are approaches which often yield results. It has been shown by Estabrook and Wahlquist (1975a) that by applying Cartan's method as well as prolongation techniques, it is possible to ascertain integrability of a given equation (Estabrook and Wahlquist, 1975b; 1976). This was accomplished for the classical Korteweg-de Vries equation. The prolongation results can be used to determine a Lax pair for the given nonlinear equation. The intention here is to develop a way to extend that work to the case of a generalized Korteweg-de Vries equation (Bracken, 2005).

Of course, the existence of an infinite number of conservation laws and the notion of integrability have both been connected to the existence of solitons associated with these equations. The study of solitons recently has been of interest due to their appearance in many physical applications (Radu and Volkov, 2008) and so the investigation of a new equation is of interest. The classical Korteweg-de Vries equation has many applications which extend beyond the original applications to solitary water waves (Bracken, 2004). It has become apparent that nonlinear dispersion can act to compatify solitary waves and generate solitons of finite wavelength. Such modes have been determined in the form of solutions to the equation which will be studied here (Bracken, 2005). The symmetry group for this equation has been determined and has allowed solutions to be calculated. These solutions include soliton solutions and solutions which can be thought of as having compact support (Rosenau and Hyman, 1993; Sivers and Takens, 1988). These equations have been of great interest recently due to their applications to areas such as coupled autonomous ocsillators and soliton theory. In the former instance, these have been a subject of interest since the discovery of their synchronization by Huygens (Pilovsky and Rosenau, 2006; Rosenau, 2006).

It is the intention here to investigate some instances of the equation which admit a larger prolongation algebra than that discussed in (Bracken, 2007) and to determine the algebra explicitly. Some of the mathematical preliminaries of Cartan's method as introduced by Estabrook and Wahlquist (1975b; 1976) are introduced first. It will be shown next how the analysis can be extended to the case of the generalized Korteweg-de Vries equation, essentially in the form given in (Bracken, 2005; 2007). An exterior differential system which reproduces the equation on the transverse manifold is presented, so integrability can be established. A prolongation is determined corresponding to this exterior differential system. Finally, a Backlund transformation is found based on the general $\mathrm{sl}(2, \mathrm{R})$ prolongation.

Exterior differential systems and Cartan prolongations: Consider the space $\mathrm{M}=\mathrm{R}^{\mathrm{n}}(\mathrm{x}, \mathrm{t}, \mathrm{u}, \mathrm{p}$, 
$\mathrm{q}, \ldots)$ in which there is defined a closed exterior differential system:

$\alpha_{1}=0 ; \ldots ; \alpha_{1}=0$

and let $\mathrm{I}$ be the ideal generated by the set $\left\{\alpha_{i}\right\}_{i=1}^{1}$ in (1) given as:

$$
I=\left\{\omega=\sum_{i=1}^{1} \sigma_{i} \wedge \alpha_{i}: \sigma_{i} \in \Lambda(M)\right\}
$$

If ideal (2) is closed, we have $\mathrm{dI} \subset \mathrm{I}$ and so (1) is integrable by a theorem. It is important to stress that system (1) is chosen such that the solutions $\mathrm{u}=\mathrm{u}(\mathrm{x}, \mathrm{t})$ of an equation:

$\mathrm{u}_{\mathrm{t}}=\mathrm{F}\left(\mathrm{x}, \mathrm{t}, \mathrm{u}, \mathrm{u}_{\mathrm{x}}, \mathrm{u}_{\mathrm{xx}}, \ldots\right)$

correspond with the two-dimensional integral manifolds of (1). There are the integral manifolds given by sections $\mathrm{S}$ of the projection:

$\pi: \mathrm{M} \rightarrow \mathrm{R}^{2}, \quad \pi(\mathrm{x}, \mathrm{t}, \mathrm{u}, \mathrm{p}, \mathrm{q}, \ldots)=(\mathrm{x}, \mathrm{t})$

These sections $\mathrm{S}$ are given by a mapping:

$S: R^{2} \rightarrow M, \quad S(x, t)=(x, t, u(x, t), p(x, t), q(x, t), \ldots)$

Introduce the fiber bundle $(\tilde{\mathrm{M}}, \tilde{\mathrm{p}}, \mathrm{M})$ over $\mathrm{M}$ with $\mathrm{M} \subset \tilde{\mathrm{M}}$ and $\tilde{\mathrm{p}}$ a projection of $\tilde{\mathrm{M}}$ onto $\mathrm{M}$, so points of $\tilde{\mathrm{M}}$ are denoted by $\tilde{\mathrm{m}}$, those in $\mathrm{M}$ by $\mathrm{m}$ and hence $(\tilde{\mathrm{p}}) \tilde{\mathrm{m}}=\mathrm{m}$.

A Cartan-Ehresmann connection in the fiber bundle $(\tilde{\mathrm{M}}, \tilde{\mathrm{p}}, \mathrm{M})$ is a system of one forms $\tilde{\omega}^{\mathrm{i}}, \mathrm{i}=1, \ldots, \mathrm{kinT}^{*}(\tilde{\mathrm{M}})$ with the property that the mapping $\tilde{\mathrm{p}}_{*}$ from the vector space:

$$
\mathrm{H}_{\tilde{\mathrm{m}}}=\left\{\tilde{\mathrm{X}} \in \mathrm{T}_{\tilde{\mathrm{m}}} \mid \tilde{\omega}^{\mathrm{i}}(\tilde{\mathrm{X}})=0, \mathrm{i}=1, \ldots, \mathrm{k}\right\}
$$

to the tangent space $\mathrm{T}_{\mathrm{m}}$ is a bijection.

We consider in $\tilde{M}$ the exterior differential system:

$$
\begin{array}{cc}
\tilde{\alpha}_{\mathrm{i}}=\tilde{p}^{*} \alpha_{\mathrm{i}}=0, & \mathrm{i}=1, \ldots, 1 \\
\tilde{\omega}^{\mathrm{j}}=0, & \mathrm{j}=1, . ., \mathrm{k}
\end{array}
$$

with $\tilde{\omega}^{\mathrm{j}}$ a Cartan-Ehresmann connection in $(\tilde{\mathrm{M}}, \tilde{\mathrm{p}}, \mathrm{M})$.

The system (6) is called a Cartan prolongation of (1) if (6) is closed and whenever $S$ is a transversal solution of (1), there should also exist a transversal local solution $\tilde{\mathrm{S}}$ of (6) with $\tilde{\mathrm{p}}(\tilde{\mathrm{S}})=\mathrm{S}$. There is a theorem which states that (6) is a Cartan prolongation of (1) if and only if (6) is closed. A necessary and sufficient condition for the existence of this is given by:

$$
\mathrm{d} \tilde{\omega}^{\mathrm{i}}=\tilde{\beta}_{\mathrm{j}}^{\mathrm{i}} \wedge \tilde{\omega}^{\mathrm{i}}, \quad \bmod \mathrm{p}^{*}(\mathrm{I})
$$

where, I is the ideal generated by $\left\{\alpha_{i}\right\}_{i=1}^{1}$ and the summation convention holds in (7). Consider a trivial bundle of the form $\tilde{M}=M \times R^{k}$ with $y=\left(y^{1}, \ldots, y^{k}\right) \in R^{k}$ and use connections which have the form:

$\tilde{\omega}^{\mathrm{i}}=\mathrm{dy}{ }^{\mathrm{i}}-\eta^{\mathrm{i}}, \quad \mathrm{i}=1, \ldots, \mathrm{k}$

with $\eta^{i}=A^{i} d x+B^{i} d t$, where $A^{i}$ and $B^{i}$ are $C^{\infty}$ functions on $\tilde{M}$. Substituting into the prolongation condition (7), it reads:

$$
-\mathrm{d} \eta^{\mathrm{i}}=\tilde{\beta}_{\mathrm{j}}^{\mathrm{i}} \wedge\left(\mathrm{dy} \mathrm{y}^{\mathrm{j}}-\eta^{\mathrm{j}}\right), \quad \bmod \tilde{\mathrm{p}}^{*}(\mathrm{I})
$$

From this, it follows that $\tilde{\beta}_{\mathrm{j}}^{\mathrm{i}}$ may be chosen such that they do not depend on $\mathrm{dy}^{\mathrm{m}}-\eta^{\mathrm{m}}, \mathrm{m}=1, \ldots, \mathrm{k}, \mathrm{m} \neq \mathrm{j}$. Moreover, it is possible to show that the prolongation condition boils down to the following:

$\mathrm{d} \eta+\frac{1}{2}[\eta, \eta]=0 \quad \bmod \tilde{\mathrm{p}}^{*}(\mathrm{I})$

Expanding out (9), the prolongation condition reads:

$(d A \wedge d x+d B \wedge d t+[A, B] d x \wedge d t)^{i} \in \tilde{p}^{*}(I)$

where, $[\mathrm{A}, \mathrm{B}]$ denotes the usual Lie-bracket of the vector fields $\mathrm{A}$ and $\mathrm{B}$.

These results can be summarized in the following theorem, which will be made use of in the following. A necessary and sufficient condition for the connection forms (8) to be a Cartan prolongation is the vanishing of its curvature form.

Cartan prolongation of the generalized Korteweg-de Vries equation: Consider the exterior differential system on the space $M$ which is defined by:

$$
\begin{aligned}
& \alpha_{1}=n u^{n-1} d u \wedge d t-p d x \wedge d t=0 \\
& \alpha_{2}=d p \wedge d t-q d x \wedge d t=0 \\
& \alpha_{3}=d u \wedge d x-d q \wedge d t-\gamma p u^{s} d x \wedge d t=0
\end{aligned}
$$


where, $\gamma$ is a constant. From system (11), we calculate that:

$$
\begin{aligned}
\mathrm{d} \alpha_{1} & =-\mathrm{dp} \wedge \mathrm{dx} \wedge \mathrm{dt}=\mathrm{dx} \wedge \alpha_{2} \\
\mathrm{~d} \alpha_{2} & =-\mathrm{dq} \wedge \mathrm{dx} \wedge \mathrm{dt}=-\mathrm{dx} \wedge \alpha_{3} \\
\mathrm{~d} \alpha_{3} & =-\gamma \mathrm{spu} u^{\mathrm{s}-1} \mathrm{du} \wedge \mathrm{dx} \wedge \mathrm{dt}-\gamma \mathrm{u}^{\mathrm{s}} \mathrm{dp} \wedge \mathrm{dx} \wedge \mathrm{dt} \\
& =\mathrm{dx} \wedge\left(\gamma \frac{\mathrm{s}}{\mathrm{n}} \mathrm{pu}^{\mathrm{s}-\mathrm{n}} \alpha_{1}+\gamma p u^{\mathrm{s}} \alpha_{2}\right)
\end{aligned}
$$

Therefore, the ideal $\mathrm{I}=\left\{\omega \mid \omega=\sum_{\mathrm{i}=1}^{3} \sigma_{\mathrm{i}} \wedge \alpha_{\mathrm{i}}: \sigma_{\mathrm{i}} \in \Lambda(\mathrm{M})\right\}$ is closed, $\mathrm{dI} \subset \mathrm{I}$ and the system $\left\{\alpha_{i}\right\}$ in (11) is integrable.

On the transversal integral manifold:

$0=\alpha_{1} \mid \mathrm{s}=\mathrm{S}^{*} \alpha_{1}=\left(\left(\mathrm{u}^{\mathrm{n}}\right)_{\mathrm{x}}-\mathrm{p}\right) \mathrm{dx} \wedge \mathrm{dt}$

$0=\alpha_{2} \mid \mathrm{s}=\mathrm{S}^{*} \alpha_{2}=\left(\mathrm{p}_{\mathrm{x}}-\mathrm{q}\right) \mathrm{dx} \wedge \mathrm{dt}$

$0=\alpha_{3} \mid \mathrm{s}=\mathrm{S}^{*} \alpha_{3}=\left(\mathrm{u}_{\mathrm{t}} \mathrm{dt} \wedge \mathrm{dx}-\mathrm{q}_{\mathrm{x}} \mathrm{dx} \wedge \mathrm{dt}-\gamma \mathrm{pu} \mathrm{s}^{\mathrm{s}} \mathrm{dx} \wedge \mathrm{dt}\right)$

The transversal integral manifolds imply the equations:

$$
p=\left(u^{n}\right)_{x}, \quad q=p_{x}\left(u^{n}\right)_{x x}, \quad u_{t}+q_{x}+\gamma p u^{s}=0
$$

Let $\mathrm{n}+\mathrm{s} \neq 0$, then substituting $\mathrm{p}$ and $\mathrm{q}$, it can be seen that $\mathrm{u}$ must satisfy the following generalized Korteweg-de Vries equation:

$$
u_{t}+\left(u^{n}\right)_{x x x}+\gamma \frac{n}{n+s}\left(u^{n+s}\right)_{x}=0
$$

This can be put in more familiar form by setting $\mathrm{m}=\mathrm{n}+\mathrm{s}$ and defining $\beta$ to be the coefficient of $\left(\mathrm{u}^{\mathrm{m}}\right)_{\mathrm{x}}$ in (14). Therefore, (14) takes the form:

$\mathrm{u}_{\mathrm{t}}+\left(\mathrm{u}^{\mathrm{n}}\right)_{\mathrm{xxx}}+\beta\left(\mathrm{u}^{\mathrm{m}}\right)_{\mathrm{x}}=0$

Using (11), the prolongation condition (10) now leads to the expression:

$A_{t} d t \wedge d x+A_{u} d u \wedge d x+A_{p} d p \wedge d x+A_{q} d q \wedge d x+$

$\mathrm{B}_{\mathrm{x}} \mathrm{dx} \wedge \mathrm{dt}+\mathrm{B}_{\mathrm{u}} \mathrm{du} \wedge \mathrm{dt}+\mathrm{B}_{\mathrm{p}} \mathrm{dp} \wedge \mathrm{dt}+\mathrm{B}_{\mathrm{q}} \mathrm{dq} \wedge \mathrm{dt}+$

$[\mathrm{A}, \mathrm{B}] \mathrm{dx} \wedge \mathrm{dt}=\lambda_{1}\left(\mathrm{nu}^{\mathrm{n}-1} \mathrm{du} \wedge \mathrm{dt}-\mathrm{pdx} \wedge \mathrm{dt}\right)+\lambda_{2}$

$(\mathrm{dp} \wedge \mathrm{dt}-\mathrm{qdx} \wedge \mathrm{dt})+\lambda_{3}\left(\mathrm{du} \wedge \mathrm{dx}-\mathrm{dq} \wedge \mathrm{dt}-\gamma \mathrm{pu}^{\mathrm{s}} \mathrm{dx} \wedge \mathrm{dt}\right)$

Comparing the coefficients of the two-forms on both sides of the prolongation condition produces the following system of equations:

$$
\begin{gathered}
-\mathrm{A}_{\mathrm{t}}+\mathrm{B}_{\mathrm{x}}+[\mathrm{A}, \mathrm{B}]=-\lambda_{1} \mathrm{p}-\lambda_{2} \mathrm{q}-\gamma \lambda_{3} \mathrm{pu}^{\mathrm{s}} \\
\mathrm{A}_{\mathrm{u}}=\lambda_{3}, \quad \mathrm{~A}_{\mathrm{p}}=0, \quad \mathrm{~A}_{\mathrm{q}}=0, \\
\mathrm{~B}_{\mathrm{u}}=\mathrm{n} \lambda_{1} \mathrm{u}^{\mathrm{n}-1}, \quad \mathrm{~B}_{\mathrm{p}}=\lambda_{2}, \quad \mathrm{~B}_{\mathrm{q}}=-\lambda_{3}
\end{gathered}
$$

To produce a small algebra, assume that $\mathrm{A}$ and $\mathrm{B}$ do not explicitly depend on $(x, t)$ so we put $A_{x}=A_{t}=0$ as well as $B_{x}=B_{t}=0$. System (16) can be put in the form:

$$
[A, B]=-\frac{1}{n} u^{-n+1} p B_{u}-q B_{p}+\gamma p u^{s} B_{q}
$$

$$
\begin{aligned}
& \mathrm{A}_{\mathrm{x}}=\mathrm{A}_{\mathrm{t}}=0 \text {, } \\
& A_{p}=0 \text {, } \\
& \mathrm{A}_{\mathrm{q}}=0, \mathrm{~A}_{\mathrm{u}}=-\mathrm{B}_{\mathrm{q}}, \quad \mathrm{B}_{\mathrm{p}}=\lambda_{2} \\
& \mathrm{~B}_{\mathrm{u}}=\mathrm{n} \lambda_{1} \mathrm{u}^{\mathrm{n}-1} \text {, }
\end{aligned}
$$

The conditions (17) imply that $\mathrm{A}=\mathrm{A}(\mathrm{u}, \mathrm{y})$ and $\mathrm{B}=$ $\mathrm{B}(\mathrm{u}, \mathrm{p}, \mathrm{q}, \mathrm{y})$. To obtain some prolongations for this system, let us take the following form for the vector field A:

$\mathrm{A}=\mathrm{A}(\mathrm{u}, \mathrm{y})=\mathrm{X}_{1}+\mathrm{u} \mathrm{X}_{2}, \mathrm{X}_{\mathrm{i}}=\mathrm{X}_{\mathrm{i}}(\mathrm{y}), \mathrm{i}=1,2$

All of the dependence on the prolongation variable $y$ resides in the $X_{i}$. Since $A_{u}=X_{2}$ from (18), then from (17) we obtain $B_{q}=-X_{2}$. This implies that $B$ is determined to be:

$\mathrm{B}=-\mathrm{q} \mathrm{X}_{2}+\mathrm{C}(\mathrm{u}, \mathrm{p}, \mathrm{y})$

Therefore, using (18) and (19), the first equation in (17) takes the form:

$$
\left[X_{1}+u X_{2},-q X_{2}+C\right]=-\frac{p}{n} u^{-n+1} C_{u}-q C_{p}-\gamma p u^{s} X_{2}
$$

Simplifying (20), it follows that:

$$
\begin{aligned}
\frac{\mathrm{p}}{\mathrm{n}} \mathrm{C}_{\mathrm{u}}+q \mathrm{qu}^{\mathrm{n}-1} \mathrm{C}_{\mathrm{p}}=- & -\gamma p \mathrm{u}^{\mathrm{s}+\mathrm{n}-1} \mathrm{X}_{2}+\mathrm{qu}^{\mathrm{n}-1}\left[\mathrm{X}_{1}, \mathrm{X}_{2}\right]- \\
& \mathrm{u}^{\mathrm{n}-1}\left[\mathrm{X}_{1}, \mathrm{C}\right]-\mathrm{u}^{\mathrm{n}}\left[\mathrm{X}_{2}, \mathrm{C}\right]
\end{aligned}
$$

Define the vector field $\mathrm{X}_{3}=\left[\mathrm{X}_{1}, \mathrm{X}_{2}\right]$, then whenever $\mathrm{C}$ is independent of $\mathrm{q}$, we obtain from (21) that $\mathrm{C}$ is given by:

$\mathrm{C}(\mathrm{u}, \mathrm{p}, \mathrm{y})=\mathrm{pX}_{3}+\mathrm{D}(\mathrm{u}, \mathrm{y})$

Substituting $\mathrm{C}$ in (22) into (21), we have: 


$$
\begin{aligned}
\frac{\mathrm{p}}{\mathrm{n}} \mathrm{D}_{\mathrm{u}}= & p\left\{-\gamma \mathrm{u}^{\mathrm{s}+\mathrm{n}-1} \mathrm{X}_{2}-\mathrm{u}^{\mathrm{n}-1}\left[\mathrm{X}_{1}, \mathrm{X}_{3}\right]-\mathrm{u}^{\mathrm{n}}\left[\mathrm{X}_{3}, \mathrm{X}_{3}\right]\right\} \\
& -\mathrm{u}^{\mathrm{n}-1}\left\{\left[\mathrm{X}_{1}, \mathrm{D}\right]-\mathrm{u}\left[\mathrm{X}_{2}, \mathrm{D}\right]\right\}
\end{aligned}
$$

Since D does not depend on p, the last term in (23) must vanish and there result two conditions on D:

$$
\begin{aligned}
& {\left[X_{1}, D\right]-u\left[X_{2}, D\right]=0} \\
& \frac{1}{n} D_{u}=\gamma u^{s+n-1} X_{2}-u^{n-1}\left[X_{1}, X_{3}\right]-u^{n}\left[X_{2}, X_{3}\right]
\end{aligned}
$$

Integrating the second equation for $\mathrm{D}$ in (24) with respect to u gives D explicitly:

$$
\begin{array}{r}
D(u, y)=-\gamma \frac{n}{m} u^{m} X_{2}-u^{n}\left[X_{1}, X_{3}\right]- \\
\frac{n}{n+1} u^{n+1}\left[X_{2}, X_{3}\right]+X_{4}
\end{array}
$$

where $\mathrm{m}=\mathrm{n}+\mathrm{s}$.

There exists a solution such that the $\mathrm{X}_{\mathrm{j}}$ satisfy the full $\mathrm{sl}(2, \mathrm{R})$ algebra, provided $\mathrm{m}, \mathrm{n}$ and $\gamma$ are interrelated in a specific way and another solution for the general case, but which satisfies a much smaller algebra. Each case will be treated in turn.

- Suppose that the $X_{j}$ satisfy the following algebra, which is isomorphic to $\mathrm{sl}(2, \mathrm{R})$ :

$$
\left[\mathrm{X}_{1}, \mathrm{X}_{2}\right]=\mathrm{X}_{3} ;\left[\mathrm{X}_{2}, \mathrm{X}_{3}\right]=2 \mathrm{X}_{2},\left[\mathrm{X}_{3}, \mathrm{X}_{1}\right]=2 \mathrm{X}_{1}
$$

Then (25) takes the form:

$$
D(u, y)=-\left(\gamma \frac{n}{m} u^{m}+2 \frac{n}{n+1} u^{n+1}\right) X_{2}+2 u^{n} X_{1}
$$

Now D in (27) must satisfy the first equation in (24) and for this to hold, we must have:

$$
\gamma \frac{\mathrm{n}}{\mathrm{m}} \mathrm{u}^{\mathrm{m}}+\frac{2 \mathrm{n}}{\mathrm{n}+1} \mathrm{u}^{\mathrm{n}+1}-2 \mathrm{u}^{\mathrm{n}+1}=0
$$

This can hold only if the powers of u match, so $m=n+1$ and if $\gamma$ is given in terms of $n$ by:

$$
\gamma=\frac{2}{n}
$$

The components of the $\eta^{\mathrm{i}}$ are then given by:

$$
\begin{aligned}
& \mathrm{A}=\mathrm{X}_{1}+\mathrm{u} \mathrm{X}_{2}, \mathrm{~B}=-\mathrm{q} \mathrm{X}_{2}+\mathrm{pX}_{3}+\mathrm{D} \\
& \mathrm{C}=\mathrm{pX}_{3}+\mathrm{d}, \mathrm{D}=-2 \mathrm{u}^{\mathrm{n}+1} \mathrm{X}_{2}+2 \mathrm{u}^{\mathrm{n}} \mathrm{X}_{1}
\end{aligned}
$$

This generates the following Lax pair:

$$
\begin{gathered}
\psi_{\mathrm{x}}=-\left(\mathrm{X}_{1}+\mathrm{uX} \mathrm{X}_{2}\right) \psi, \\
\psi_{\mathrm{t}}=-\left[2 \mathrm{u}^{\mathrm{n}}-\left(2 \mathrm{u}^{\mathrm{n}+1}+q\right) \mathrm{X}_{2}+\mathrm{pX_{3 }}\right] \psi
\end{gathered}
$$

Where:

$$
\mathrm{p}=\left(\mathrm{u}^{\mathrm{n}}\right)_{\mathrm{x}}, \mathrm{q}=\mathrm{p}_{\mathrm{x}}
$$

- Let us find another solution to (24) such that m, n and $\gamma$ are arbitary. No assumptions whatever are made with regard to these parameters. Substituting D from (25) into the first Eq. 24 gives:

$$
\begin{aligned}
& -\gamma \frac{\mathrm{n}}{\mathrm{m}} \mathrm{u}^{\mathrm{m}}\left[\mathrm{X}_{1}, \mathrm{X}_{2}\right]+\mathrm{u}^{\mathrm{n}}\left[\mathrm{X}_{1},\left[\mathrm{X}_{1}, \mathrm{X}_{3}\right]\right]+\left[\mathrm{X}_{1}, \mathrm{X}_{4}\right] \\
& -\mathrm{u}^{\mathrm{n}+1}\left\{\frac{\mathrm{n}}{\mathrm{n}+1}\left[\mathrm{X}_{1},\left[\mathrm{X}_{2}, \mathrm{X}_{3}\right]\right]+\left[\mathrm{X}_{2}\left[\mathrm{X}_{1}, \mathrm{X}_{3}\right]\right]\right\} \\
& +\frac{\mathrm{n}}{\mathrm{n}+1} \mathrm{u}^{\mathrm{n}+2}\left[\mathrm{X}_{2},\left[\mathrm{X}_{2}, \mathrm{X}_{3}\right]\right]-\mathrm{u}\left[\mathrm{X}_{2}, \mathrm{X}_{4}\right]=0
\end{aligned}
$$

There is not much freedom in balancing powers of $\mathrm{u}$ in (29). To satisfy (29) if no assumptions are made with regard to $m$ and $n$, we must require that:

$$
\left[\mathrm{X}_{1}, \mathrm{X}_{4}\right]=0,\left[\mathrm{X}_{2}, \mathrm{X}_{4}\right]=0
$$

To satisfy these brackets, this can be done in the following way. Let us take $\mathrm{X}_{4}=\mu \mathrm{X}_{2}$ and $\mathrm{X}_{4}=\kappa \mathrm{X}_{1}$, from which it follows that $X_{1}=\lambda X_{2}$ and $\mu, \kappa$ and $\lambda$ are real constants. Moreover, substituting these results into the definition of $X_{3}$, it follows that $X_{3}=0$. Using all of these results in (29), it follows that the remaining terms in (29) vanish. Hence (29) is satisfied identically and we have one solution. To summarize these results for the vector fields, we have:

$X_{1}=\lambda X_{2}, \quad X_{2}, \quad X_{3}=0, \quad X_{4}=\mu X_{2}$

The prolongation structure reduces to the following set of vector fields:

$$
\begin{aligned}
& \mathrm{A}=(\lambda+u) X_{2} \\
& \mathrm{~B}=\mathrm{q} X_{2}+\mathrm{C}=\left(-\mathrm{q}-\gamma \frac{\mathrm{n}}{\mathrm{m}} \mathrm{n}^{\mathrm{m}}+\mu\right) \mathrm{X}_{2} \\
& \mathrm{C}=\mathrm{D}=\left(-\gamma \frac{\mathrm{n}}{\mathrm{m}} \mathrm{u}^{\mathrm{m}}+\mu\right) \mathrm{X}_{2}, \quad \lambda, \mu \in \mathrm{R}
\end{aligned}
$$

Consider $\mathrm{X}_{2}$ to be one of the generators of $\mathrm{sl}(2, \mathrm{R})$, so the solution (32) is based on a subalgebra.

It can be represented in matrix form as: 
$\mathrm{X}_{1}=\frac{1}{2}\left(\begin{array}{cc}0 & -1 \\ -1 & 0\end{array}\right), \mathrm{X}_{2}=\frac{1}{2}\left(\begin{array}{cc}0 & 1 \\ -1 & 0\end{array}\right), \mathrm{X}_{3}=\frac{1}{2}\left(\begin{array}{cc}1 & 0 \\ 0 & -1\end{array}\right)$

To find the Maurer-Cartan algebra of $\mathrm{GL}(n, R)$, we consider the left-invariant forms $\omega_{i}^{j}$ as elements of a matrix:

$\omega-\left(\omega_{i}^{j}\right)-Y^{-1} d Y$

where, $\mathrm{Y}$ is the natural embedding of the group into $\mathrm{R}^{\mathrm{n}^{2}}$. Then $\mathrm{Y}^{-1} \mathrm{dY}$ is the Maurer-Cartan form. The Maurer-Cartan algebra can be written as:

$\mathrm{d} \omega+\omega \wedge \omega=0$

In this case we take $\mathrm{SL}(2, \mathrm{R})=\{\mathrm{X} \in \mathrm{GL}(2, \mathrm{R}) \operatorname{det}(\mathrm{X})=1\} . \quad$ The exponential map can be used to obtain the Maurer-Cartan algebra. To obtain a form for (34) that is more convenient, weintroduce the $\omega^{\mathrm{i}}$ by:

$\omega=\left(\begin{array}{cc}\omega_{1}^{1} & \omega_{1}^{2} \\ \omega_{2}^{1} & -\omega_{1}^{1}\end{array}\right)=\frac{1}{2}\left(\begin{array}{cc}\omega^{1} & \omega^{3}-\omega^{2} \\ -\omega^{3}-\omega^{2} & -\omega^{1}\end{array}\right)$

Substituting (35) into (34), it follows that the $\omega^{\mathrm{i}}$ satisfy the Maurer-Cartan relations:

$\mathrm{d} \omega^{1}=\omega^{3} \wedge \omega^{2}, \mathrm{~d} \omega^{2}=\omega^{1} \wedge \omega^{3}, \mathrm{~d} \omega^{3}=\omega^{1} \wedge \omega^{2}$

Using (32), we calculate A and B to be:

$$
\begin{aligned}
& A=\frac{1}{2}\left(\begin{array}{cc}
0 & \lambda+u \\
-\lambda-u & 0
\end{array}\right), \\
& B=\frac{1}{2}\left(\begin{array}{cc}
0 & -q-\gamma \frac{n}{m} u^{m}+\mu \\
q+\gamma \frac{n}{m} u^{m}-\mu & 0
\end{array}\right)
\end{aligned}
$$

and the cocycle is given by:

$$
\begin{aligned}
& \sigma=\frac{1}{2}\left(\begin{array}{cc}
0 & \lambda+u \\
-\lambda-u & 0
\end{array}\right) \\
& d x+\frac{1}{2}\left(\begin{array}{cc}
0 & -q-\gamma \frac{n}{m} u^{m}+\mu \\
q+\gamma \frac{n}{m} u^{m}-\mu & 0
\end{array}\right) d t
\end{aligned}
$$

If we let the Maurer-Cartan form have the structure (35), then the $\sigma^{\mathrm{i}}$ are found to be: $\sigma^{1}=0, \quad \sigma^{2}=0$,
$\sigma^{3}=\frac{1}{2}(\lambda+u) d x-\frac{1}{2}\left(q+\gamma \frac{n}{m} u^{m}-\mu\right) d t$

System (39) satisfies the Maurer-Cartan algebra if and only if $u$ satisfies (14 and 15). The group $\operatorname{SL}(2, \mathrm{R})$ can be written as a product:

$$
\begin{gathered}
\mathrm{SL}(2, \mathrm{R})=\mathrm{BL}(2, \mathrm{R}) \cdot \mathrm{SO}(2) \\
\mathrm{Y}=\mathrm{A} \cdot \mathrm{B}
\end{gathered}
$$

Hence the Maurer-Cartan form is:

$\omega=B^{-1}\left(A^{-1} d A\right) \cdot B+B^{-1} d B$

where, $\mathrm{A}$ and $\mathrm{B}$ are:

$A=\left(\begin{array}{cc}a & 0 \\ b & \frac{1}{a}\end{array}\right), \quad B=\left(\begin{array}{cc}\cos \beta & \operatorname{xin} \beta \\ -\sin \beta & \cos \beta\end{array}\right)$

Introducing the forms:

$\tau^{1}=\frac{2}{\mathrm{a}} \mathrm{da}, \quad \tau^{2}=\mathrm{bda}-\mathrm{adb}, \quad \psi=2 \beta$

$\omega$ can be written as:

$$
\left(\begin{array}{c}
\omega^{1} \\
\omega^{2}
\end{array}\right)=\left(\begin{array}{cc}
\cos \psi & \sin \psi \\
-\sin \psi & \cos \psi
\end{array}\right)\left(\begin{array}{c}
\tau^{1} \\
\tau^{2}
\end{array}\right), \quad \omega^{3}=\tau^{2}+\mathrm{d} \psi
$$

From the function $f: R^{2} \omega f: R^{2} \rightarrow \operatorname{SL}(2, R)$ with $f^{*}\left(\omega^{i}\right)=\sigma^{\mathrm{i}}$, it follows that:

$\left(\begin{array}{c}\tilde{\sigma}^{1} \\ \tilde{\sigma}^{2}\end{array}\right)=\left(\begin{array}{cc}\cos \psi & -\sin \psi \\ \sin \psi & \cos \psi\end{array}\right)\left(\begin{array}{c}\sigma^{1} \\ \sigma^{2}\end{array}\right), \quad \quad \tilde{\sigma}^{3}=\sigma^{3}+\mathrm{d} \psi$

Solving (44) for d, we get:

$\mathrm{d} \psi=\sigma^{3}-\sin \psi \sigma^{1}-\cos \psi \sigma^{2}$ by:

Putting (49) into (45), it follows that $\mathrm{d} \psi$ is given $\mathrm{d} \psi=\frac{1}{2}(\lambda+\mathrm{u}) \mathrm{dx}-\frac{1}{2}\left(\mathrm{q}+\gamma \frac{\mathrm{n}}{\mathrm{m}} \mathrm{u}^{\mathrm{m}}-\mu\right) \mathrm{dt}$

From (46), a Backlund transform can be extracted: 
$\psi_{\mathrm{x}}=\frac{1}{2}(\lambda+\mathrm{u}), \quad \psi_{\mathrm{t}}=-\frac{1}{2}\left(\left(\mathrm{u}^{\mathrm{n}}\right)_{\mathrm{xx}}+\gamma \frac{\mathrm{n}}{\mathrm{m}} \mathrm{u}^{\mathrm{m}}-\mu\right)$

To obtain the equation in terms of which goes along with (47), solve the first equation in (47) for $u$ and then substitute $u$ into the second equation of (47). Upon doing this, we find that $\mathrm{u}=2 \psi_{\mathrm{x}}-\lambda$ and:

$$
\psi_{\mathrm{t}}=-\frac{1}{2}\left(\left(\left(2 \psi_{\mathrm{x}}-\lambda\right)^{\mathrm{n}}\right)_{\mathrm{xx}}+\gamma \frac{\mathrm{n}}{\mathrm{m}}\left(2 \psi_{\mathrm{x}}-\lambda\right)^{\mathrm{m}}-\mu\right)
$$

\section{CONCLUSION}

It has been shown that by applying the general method of Estabrook and Wahlquist (1975a), the integrability of a generalized Korteweg-de Vries Eq. 14 can be established. The prolongation structures that are produced can be used to give a type of Lax pair for the system.

\section{REFERENCES}

Bracken, P., 2004. Some methods for generating solutions to the Korteweg-de Vreis equation. Phys. A., 335: 70-78.

Bracken, P., 2005. Symmetry properties of a generalized Korteweg-de Vries equation and some explicit solutions. Int. J. Math. Math. Sci., 15: 2159-2173.

Bracken, P., 2007. An exterior differential system for a generalized Korteweg-de Vries equation and its associated integrability. Acta Applied Math., 95: 223-231.

Das, A., 1989. Integrable models. World Sci. Lecture Notes Phys., 30.
Estabrook, F.B. and H.D. Wahlquist, 1975a. The geometric approach to sets of ordinary differential equations and Hamiltonian dynamics. Siam Rev., 17: 201-220.

Estabrook, F.B. and H.D. Wahlquist, 1975b. Prolongation structure of nonlinear evolution equations. J. Math. Phys., 16: 1-7.

Estabrook, F.B. and H.D. Wahlquist, 1976. Prolongation structures of nonlinear evolution equations II. J. Math., 17: 1293-1297.

Pilovsky, A. and P. Rosenau, 2006. Phase compactons. Phys. D., 218: 56-69.

Radu, E. and M.S. Volkov, 2008. Stationary ring solitons in field theory-Knots and vortons. Phys. Rep., 468: 101-151.

Rosenau, P. and J. Hyman, 1993. Compactons: Solitons with finite wavelength. Phys. Rev. Lett., 70: 564-567.

Rosenau, P., 2006. On a model equation of travelling and stationary compactons. Phys. Lett. A., 256: 44-50.

Sivers, A.J. and S. Takens, 1988. Intrinsic localized modes in anharmonic crystals. Phys. Rev. Lett., 61: 970-973. 